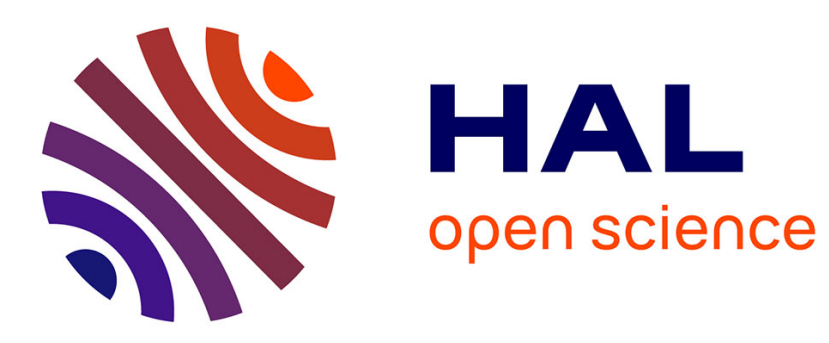

\title{
Probing turbulence intermittency via autoregressive moving-average models
}

Davide Faranda, Bérengère Dubrulle, François Daviaud, Flavio Maria, Emanuele Pons

\section{To cite this version:}

Davide Faranda, Bérengère Dubrulle, François Daviaud, Flavio Maria, Emanuele Pons. Probing turbulence intermittency via autoregressive moving-average models. Physical Review E: Statistical, Nonlinear, and Soft Matter Physics, 2014, 90, pp.061001(R). 10.1103/PhysRevE.90.061001 . cea01370472

\section{HAL Id: cea-01370472 https://hal-cea.archives-ouvertes.fr/cea-01370472}

Submitted on 22 Sep 2016

HAL is a multi-disciplinary open access archive for the deposit and dissemination of scientific research documents, whether they are published or not. The documents may come from teaching and research institutions in France or abroad, or from public or private research centers.
L'archive ouverte pluridisciplinaire $\mathbf{H A L}$, est destinée au dépôt et à la diffusion de documents scientifiques de niveau recherche, publiés ou non, émanant des établissements d'enseignement et de recherche français ou étrangers, des laboratoires publics ou privés. 


\title{
Probing turbulence intermittency via autoregressive moving-average models
}

\author{
Davide Faranda, ${ }^{*}$ Bérengère Dubrulle, and François Daviaud \\ Laboratoire SPHYNX, Service de Physique de l'Etat Condensé, DSM, CEA Saclay, CNRS URA No. 2464, 91191 Gif-sur-Yvette, France \\ Flavio Maria Emanuele Pons \\ Department of Statistics, University of Bologna, Via delle Belle Arti 41, 40126 Bologna, Italy \\ (Received 4 July 2014; published 3 December 2014)
}

\begin{abstract}
We suggest an approach to probing intermittency corrections to the Kolmogorov law in turbulent flows based on the autoregressive moving-average modeling of turbulent time series. We introduce an index $\Upsilon$ that measures the distance from a Kolmogorov-Obukhov model in the autoregressive moving-average model space. Applying our analysis to particle image velocimetry and laser Doppler velocimetry measurements in a von Kármán swirling flow, we show that $\Upsilon$ is proportional to traditional intermittency corrections computed from structure functions. Therefore, it provides the same information, using much shorter time series. We conclude that $\Upsilon$ is a suitable index to reconstruct intermittency in experimental turbulent fields.
\end{abstract}

DOI: 10.1103/PhysRevE.90.061001

PACS number(s): 47.27.E-

Introduction. One of the few exact results known for isotropic, homogeneous, and mirror-symmetric turbulence is the 4/5 law derived by Kolmogorov. It links the longitudinal velocity increments $\delta u_{\ell}=u(x+\ell)-u(x)$ to the mean rate of energy dissipation $\langle\epsilon\rangle$ via

$$
\left\langle\delta u_{\ell}^{3}\right\rangle=-\frac{4}{5}\langle\epsilon\rangle \ell
$$

where angular brackets denote averaging. This exact relation was generalized by Kolmogorov [1] as a scaling law $\delta u_{\ell} \equiv$ $(\epsilon \ell)^{1 / 3}$, where $\equiv$ means has the same statistical properties. Should $\epsilon$ be a nonstochastic constant, the scaling law would imply self-similar behavior for the structure functions of order $p, S_{p}(\ell)=\left\langle\delta u_{\ell}^{p}\right\rangle$, which would scale like

$$
F_{p}(\ell) \sim \epsilon^{p / 3} \ell^{p / 3} \text {. }
$$

For $p=3$, we recover the $4 / 5$ law. For $p=2$, this equation predicts a second-order structure function that varies like $\ell^{2 / 3}$. By a Fourier transform, this is equivalent to a one-dimensional energy spectrum scaling with wave number $k$ as $E(k) \sim k^{-5 / 3}$, also known as the Kolmogorov spectrum [2,3]. Both the 4/5 law and the Kolmogorov spectrum have been measured and checked in many natural and laboratory isotropic turbulent flows [4]. More generally, Eq. (2) predicts a linear law for the exponent of the structure functions $\zeta(p)=d \ln F_{p}(\ell) / d \ln \ell=$ $p / 3$. However, as pointed out by Landau and recognized by Kolmogorov [1], there is no reason to assume that $\epsilon$ is a constant over space and/or time, so it should rather be viewed as a stochastic process, which depends upon the scale $\ell$ at which it is measured $\epsilon \equiv \epsilon(\ell)$. In such a case, the correct scaling of the structure function is rather

$$
F_{p}(\ell) \sim\left\langle\epsilon(\ell)^{p / 3}\right\rangle \ell^{p / 3} .
$$

This modified law predicts correction to the linear law $\zeta(p)=p / 3$, which is connected to the intermittent nature of the dissipation. For example, a log-normal model for the dissipation (a suggestion by Landau and Obukhov) implies

\footnotetext{
*davide.faranda@cea.fr
}

quadratic corrections for the $\zeta(p)$. Other models have been suggested and lead to different corrections [5-7]. Intermittency corrections up to $p=4$ have been measured in a variety of experimental and numerical flows and appear to be robustly consistent from one experiment to another (see, e.g., the review of [8]). Corrections for larger values of $p$ are subject to resolution and statistical convergence issues: The larger the scaling exponent, the larger the statistical sampling must be in order to capture the rare events. There is presently no general consensus about the behavior of intermittency corrections at large order. This hinders progress in the understanding of the statistical properties of the energy dissipation. In this Rapid Communication we suggest an approach to probing intermittency corrections based on the autoregressive movingaverage (ARMA) modeling of turbulent time series. We introduce an index $\Upsilon$ that measures the distance from a Kolmogorov-Obukhov model in the ARMA space. Applying our analysis to velocity measurements in a von Kármán swirling flow, we show that this index is proportional to the traditional intermittency correction computed from the structure function and provides the same information, using shorter time series.

Intermittency parameters. In most laboratory turbulent flows, data sets are time series of values of a physical observable at a fixed point or obtained by tracking Lagrangian particles. This motivated the shift of paradigm from space velocity increments to time velocity increments defined as $\delta u_{\tau}=u(t+\tau)-u(t)$ and motivated measurements of time structure functions $G_{p}(\tau)=\left\langle\left(\delta u_{\tau}\right)^{p}\right\rangle$ and its local exponent $\chi_{p}=d \ln G_{p}(\tau) / d \ln \tau$. In situations where measurements are made on the background of a strong mean velocity $U$, scale velocity increments and time velocity increments can be directly related through the Taylor hypothesis $\ell=U \tau$. When fluctuations are of the same order as the mean flow, however, the Taylor hypothesis fails. A suggestion has been made in [9] to then resort to a local Taylor hypothesis, in which $\ell=\int d t u(t)$, where $u$ is the local rms velocity. This is equivalent to considering a scale such that $\ell \sim \tau \delta u_{\tau}$ and may be seen as equivalent to modifying the space-refined Kolmogorov hypothesis into a time hypothesis $\delta u_{\tau} \equiv(\epsilon \tau)^{1 / 2}$, 
which leads to

$$
G_{p}(\tau) \sim\left\langle\epsilon^{p / 2}\right\rangle \tau^{p / 2}
$$

Such scaling is equivalent to the scaling obtained using the Lagrangian structure function. In any case, we may define the intermittency as the deviation of the local exponents $\zeta_{p}^{*}=\zeta_{p}$ (space increments) or $\zeta_{p}^{*}=\chi_{p}$ (time increments) with respect to a linear behavior and may be quantified to first order by the parameter

$$
\mu=\zeta_{2}^{*}-\frac{2}{3} \zeta_{3}^{*} .
$$

This factor is proportional to the logarithm of the $\beta$ parameter of the log-Poisson model [5,6], or to the $\mu$ parameter of the lognormal model [1]. It is also valid when the scaling exponents have been computed using extended self-similarity (ESS) [10], which is especially interesting in situations where turbulence is inhomogeneous and when the Taylor hypothesis does not hold. In the following, we compare this intermittency index with another one, built in a purely statistical framework.

Indeed, Thomson [11] showed that, in the Lagrangian framework, the time-refined Kolmogorov hypothesis is in fact equivalent to a stochastic description in terms of an Ornstein-Uhlenbeck process with suitable drift and noise term

$$
d u=-\frac{u}{T} d t+\sqrt{C_{0} \epsilon} d W,
$$

where $T$ is a decorrelation time scale, $C_{0}$ is a universal constant, and $\epsilon$ is the mean dissipation. Indeed, taking into account the definition of the particle position $x, d x=u d t$, we get a scaling of the time averages of velocity and position as

$$
\overline{u^{2}(t)} \sim t, \quad \overline{x^{2}(t)} \sim t^{3} .
$$

The second property is the Richardson law. Then, defining $\delta u=\left[\overline{u^{2}(t)}\right]^{1 / 2}$ and $\ell=\left[\overline{x^{2}(t)}\right]^{1 / 2}$, we get from Eq. (7) $\delta u \sim$ $\ell^{1 / 3}$, which leads to the space-refined Kolmogorov hypothesis.

The discrete-time version of Eq. (6) can be written as

$$
u_{t}=\phi u_{t-1}+\psi_{t}
$$

where $t$ is a discrete-time label, $d W$ are the increments of a Brownian motion, $\phi=\left(1-\frac{\Delta t}{T}\right)$, and $\psi_{t}$ are independent variables, normally distributed. Equation (8) is the expression of an autoregressive process of order one, denoted by $\operatorname{AR}(1)$. Such a model is described by a single decorrelation time. So it cannot describe real flows intermittency, which involves a whole range of time scales corresponding to the turnover times of the turbulent eddies with memory effects. To capture these effects, it is mandatory to consider a projection of the velocity data on higher-order $\operatorname{ARMA}(p, q)$ models. This enables a quantification of intermittency effects as a distance with respect to the insufficient AR(1) model in this space.

Intermittency as a distance in ARMA space. A stationary time series $X_{t}$ is said to follow an $\operatorname{ARMA}(p, q)$ process if it satisfies the discrete equation

$$
X_{t}=\sum_{i=1}^{p} \phi_{i} X_{t-i}+\varepsilon_{t}+\sum_{j=1}^{q} \theta_{j} \varepsilon_{t-j},
$$

with $\varepsilon_{t} \sim N_{W}\left(0, \sigma^{2}\right)$, where $N_{W}$ stands for white noise and the polynomials $\phi(z)=1-\phi_{1} z_{t-1}-\cdots-\phi_{p} z_{t-p}$ and $\theta(z)=$ $1-\theta_{1} z_{t-1}-\cdots-\theta_{q} z_{t-q}$, with $z \in \mathbb{C}$, have no common factors. Notice that the noise term $\varepsilon_{t}$ will be assumed to be a white noise, which is a general condition [12]. We ensure unicity by applying the Box-Jenkis procedure [13]: We choose the lowest $p$ and $q$ such that the residuals of the series filtered by the process $\operatorname{ARMA}(p, q)$ are not correlated. To define a suitable distance in the space of $\operatorname{ARMA}(p, q)$ models, we introduce the Bayesian information criterion $C_{B I}$, measuring the relative quality of a statistical model, as

$$
C_{B I}=-2 \ln \hat{L}\left(n, \hat{\sigma}^{2}, p, q\right)+k[\ln (n)+\ln (2 \pi)],
$$

where $\hat{L}\left(n, \hat{\sigma}^{2}, p, q\right)$ is the likelihood function for the investigated model, $k=p+q$, and $n$ is the length of the sample. The variance $\hat{\sigma}^{2}$ is computed from the sample and is a series-specific quantity. The normalized distance between the fit $\operatorname{ARMA}(p+1, q)$ and the Kolmogorov AR(1) model is then defined as the normalized difference between the $C_{B I}\left(n, \hat{\sigma}^{2}, p+1, q\right)$ and the $\operatorname{AR}(1) C_{B I}\left(n, \hat{\sigma}^{2}, 1,0\right)$ :

$$
\Upsilon=1-\exp \left\{\left|C_{B I}(p+1, q)-C_{B I}(1,0)\right|\right\} / n .
$$

The $p+1$ serves to magnify $\Upsilon$ near zero. Note that $0 \leqslant \Upsilon \leqslant$ 1: It goes to zero if the data set is well described by an $\operatorname{AR}(1)$ model and tends to one in the opposite case. In the case of velocity increments time series, it measures deviations from the Kolmogorov model.

Application to turbulent data. We apply the index defined in Eq. (11) to velocity time series obtained in a von Kármán turbulent swirling flow. The experimental setup consists of two sets of blades mounted on two counterrotating coaxial impellers at the top and bottom of a cylindric vessel of diameter $R=0.1 \mathrm{~m}$. The operating fluid is water and the rotation frequency of the impellers can reach $F=15 \mathrm{~Hz}$, resulting in large Reynolds numbers $\left(\operatorname{Re}=2 \pi F R^{2} v^{-1} \sim 10^{6}\right)$. A detailed description of the experiment can be found in [14-16]. Two techniques are used to measure the fluid velocity on a grid: the particle interferometry velocimetry (PIV) and the laser Doppler velocimetry (LDV), mapped on a regular sampling time applying a sample-and-hold algorithm. The stereoscopic PIV measures the three components of the velocity field in a plane, while the LDV measurements provide the out-of-plane velocity component $V_{\phi}$ in a plane. The PIV produces regularly sampled time series at intervals of $0.1 \mathrm{~s}$ over a sample size at most of order $10^{4}$ and a spatial resolution of the order of $1 \mathrm{~mm}$, i.e., 10-100 times larger than the dissipation scale. The LDV time series are sampled over a time scale of the order of $0.001 \mathrm{~s}$, producing a sample size up to $10^{6}$ data on a grid of spatial resolution of the order of $1 \mathrm{~cm}$. Given these resolution constraints, we compute spatial (temporal) velocity increments for the PIV (LDV) data. The idea is to compute at each spatial grid location the classical intermittency index $\mu$, compare it to $\Upsilon$, and see how they vary. All the analyses presented in this Rapid Communication are done using three components for the PIV and $V_{\phi}$ for the LDV. Since the von Kármán flow is inhomogeneous and anisotropic with large fluctuations [16], we expect that the time and space velocity structure functions depend on the measurement points. This is illustrated in Fig. 1 for the second- and fourth-order spatial and time structure functions. For the spatial case, deviations from the Kolmogorov scaling (solid lines) are small for the spatial structure functions, near the symmetry plane $Z=0$. This plane 

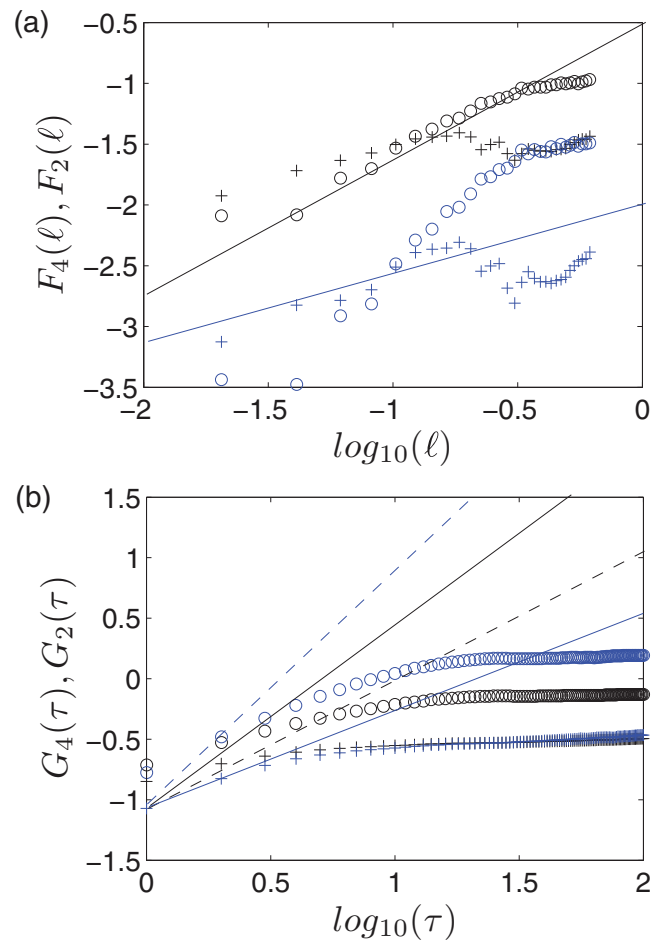

FIG. 1. (Color online) (a) Space structure function $F_{p}(\ell)$ of order 2 in black and of order 4 in blue (gray) at two PIV grid points of coordinates $R=-0.10, Z=-0.14$ (circles) and $R=0.98, Z=$ 0.70 (crosses). (b) Time structure function $G_{p}(\tau)$ of order 2 in black and of order 4 in blue (gray) at two LDV grid points of coordinates $R=0, Z=0.35$ (circles) and $R=0.52, Z=0.61$ (crosses). Lines represent the Kolmogorov predictions: solid is Eulerian with $\ell^{2 / 3}$ and $\tau^{2 / 3}$ in black and $\ell^{4 / 3}$ or $\tau^{4 / 3}$ in blue (gray) and dotted is Lagrangian with $\tau$ in black and $\tau^{2}$ in blue (gray).

is the location of an intense shear layer and has traditionally been used to perform isotropic homogeneouslike measurements. Outside this plane, deviations from the Kolmogorov scaling are large. For the time case, one observes two distinct behaviors: Outside the shear layer, where a mean velocity is well defined, one observes close to Eulerian-Kolmogorov scaling at the smallest time increments $\tau^{p / 3}$; in the shear layer, where no Taylor hypothesis holds, the scaling is closer to Lagrangian scaling $\tau^{p / 2}$. However, as already noted by [9] and shown in Fig. 2, the relative scaling exponents $\zeta_{p}^{*}$ computed as $G_{p}(\tau) \sim\left\langle\left|\delta u_{\tau}\right|^{3}\right\rangle^{\zeta_{p}^{*}}$ (ESS method) are in most of the flow close to the universal scaling exponents found by [8], in a variety of homogeneous turbulent flows, even those with no obvious inertial range. Using these ESS scaling exponents to compute the $\mu$ index, we may then draw a map of the intermittency and compare it with $\Upsilon$. This is done in Fig. 3 for an LDV experiment at $\operatorname{Re} \sim 10^{5}$. The spatial patterns look indeed similar. Moreover, the plot of $\Upsilon$ as a function of $\mu$ [Fig. 3(c)] evidences a linear relation between them; the linear regression represented by the red line leads to a linear correlation coefficient $r \simeq 0.69$. This means that $\Upsilon$ traces the same intermittency characteristics as the time structure functions. The comparison of $\Upsilon$ with the intermittency index $\mu$ computed for spatial structure functions is also informative: Because of convergency issues, we have to use a data set

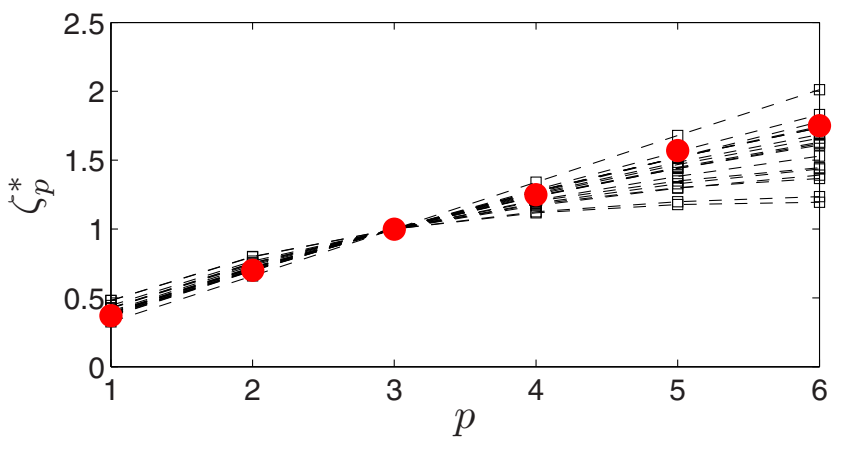

FIG. 2. (Color online) Plot of $\zeta_{p}^{*}$ computed for the LDV experiments. Different lines correspond to different measure points. Red (gray) spots mark the scaling exponents reported in [8].

of about $10^{5}-10^{6}$ data points to converge the estimate of $\mu$, while only $10^{3}$ are needed to converge $\Upsilon$. To illustrate this, we use the longest data set available: 9000 velocity fields of a PIV experiment performed at $\mathrm{Re} \simeq 5 \times 10^{4}$. At this value, the von Kármán flow experiences the equivalent of a phase transition [17], with time wandering of the shear layer in between $Z=0.3$ and -0.3 . This corresponds to a very large time intermittency and is detected by the $\Upsilon$ index as shown in Fig. 4, under the shape of two patches at $R \simeq 0$, $Z=0.3$ and $R \simeq 0, Z=-0.3$. This pattern is unique to the phase transition and is not present in other PIV experiments [18]. Besides, one observes a fairly symmetric structure, with maxima corresponding to the flow's four-cell structure. The time intermittency prevents the convergence of the spatial

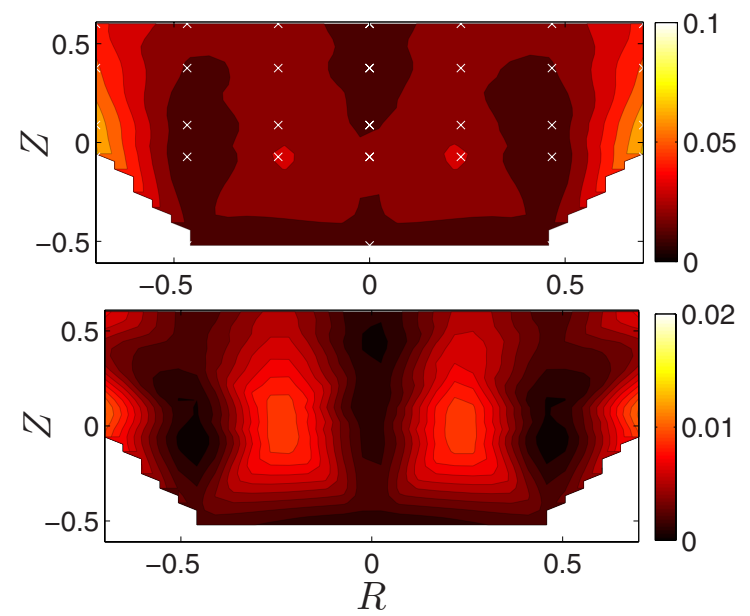

(a)

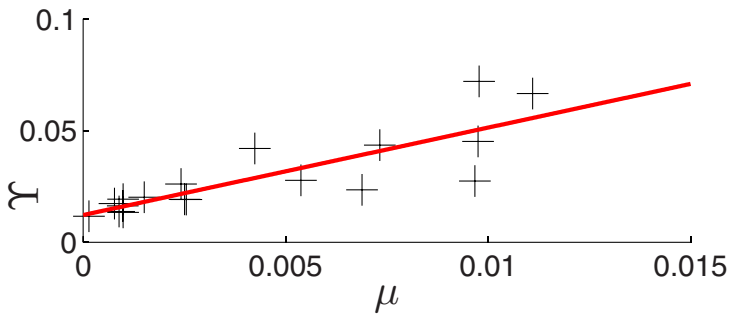

(c)

FIG. 3. (Color online) (a) Index $\Upsilon$ vs (b) the intermittency index $\mu=\zeta_{2}^{*}-\frac{2}{3} \zeta_{3}^{*}$. White crosses show measurement points. (c) Scatter plot of $\Upsilon$ vs $\mu$. The red (gray) line shows a linear regression of the data. 

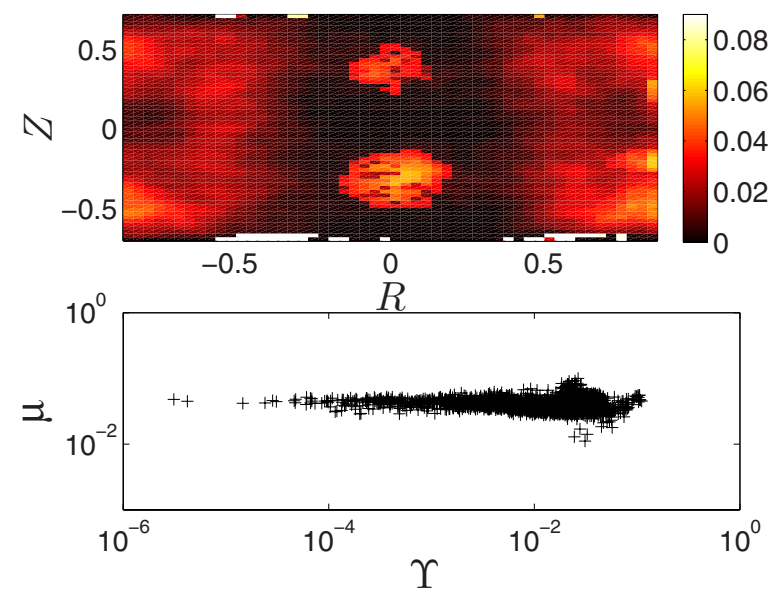

(b)

FIG. 4. (Color online) (a) Index $\Upsilon$ computed for a PIV experiment. The intermittency $\Upsilon$ maxima are localized near the walls and trace the position of the time wandering shear layer at $R=0$. (b) Scatter plot of $\Upsilon$ vs $\mu$, the former displaying a much greater sensitivity than the traditional intermittency index, despite the short length of the time series.

structure functions, resulting in a lack of symmetry of the $\mu$ field (not shown). As a result, $\mu$ fluctuates over a decade around a value of about 0.05 , while $\Upsilon$ spans several orders of magnitude, as can be seen in Fig. 4(b). This shows that $\Upsilon$ is a more sensitive tool to detect intermittency than $\mu$.

Discussion. We have introduced an intermittency index $\Upsilon$ that can be interpreted as a statistical distance between the best-fit linear ARMA model for a turbulent time series and the simplest possible process, i.e., AR(1). We have compared such an index with a classical intermittency index $\mu=\zeta_{2}^{*}-\frac{2}{3} \zeta_{3}^{*}$. In statistically converged LDV series, the two parameters are linearly related, with a regression coefficient $R \simeq 0.69$. In shorter PIV time series, $\Upsilon$ catches important characteristics of the mean flow in situations where $\mu$ cannot be computed due to a lack of convergence. Therefore, the main advantage of this index is the applicability to cases in which no big data sets are available. Moreover, ARMA models contain the information on nonlocal interactions between large and small scales in the parameters $\phi_{i}$ and $\theta_{i}$. In general, the $\operatorname{AR}(p)$ part of an $\operatorname{ARMA}(p, q)$ process is the contribution of the large scales and represents the persistence of the process. If the process is more persistent than $\operatorname{AR}(1)$ with $|\phi|<1$, higher values of $p$ are required to explain all the correlation coming from the large scales. Analogous considerations hold for the $\operatorname{MA}(q)$ part. When $\Phi \ll \Theta$ there is a clear separation between large and small scales. On the other hand, when their magnitude is similar such separation is not ensured anymore [18]. Our results reinforce the hypothesis of [19] that intermittency propagates in direct interactions between large and small scales, rather than in cascades. Finally, our method can be used to validate models based on the stochastic differential equation [20] with respect to experimental data.

Acknowledgments. Experimental data were provided with the help of E. Herbert, P. Ph. Cortet, C. Wiertel, and V. Padilla. D.F. acknowledges the support of a Centre National de la Recherche Scientifique postdoctoral grant.
[1] A. N. Kolmogorov, J. Fluid Mech. 13, 82 (1962).

[2] A. N. Kolmogorov, Dokl. Akad. Nauk SSSR 30, 299 (1941).

[3] A. Obukhov, Dokl. Akad. Nauk SSSR 32, 22 (1941).

[4] U. Frisch, Turbulence (Cambridge University Press, Cambridge, 1996), Vol. 1, p. 310.

[5] Z.-S. She and E. Leveque, Phys. Rev. Lett. 72, 336 (1994).

[6] B. Dubrulle, Phys. Rev. Lett. 73, 959 (1994).

[7] R. Benzi, G. Paladin, G. Parisi, and A. Vulpiani, Phys. Rev. Lett. 17, 3521 (1984).

[8] A. Arneodo, C. Baudet, F. Belin, R. Benzi, B. Castaing, B. Chabaud, R. Chavarria, S. Ciliberto, R. Camussi, F. Chilla et al., Europhys. Lett. 34, 411 (1996).

[9] J.-F. Pinton and R. Labbé, J. Phys. II 4, 1461 (1994).

[10] R. Benzi, S. Ciliberto, C. Baudet, G. R. Chavarria, and R. Tripiccione, Europhys. Lett. 24, 275 (1993).

[11] D. J. Thomson, J. Fluid Mech. 180, 529 (1987).

[12] P. J. Brockwell and R. A. Davis, Time Series: Theory and Methods, 2nd ed. (Springer, Berlin, 1990).
[13] G. E. Box, G. M. Jenkins, and G. C. Reinsel, Time Series Analysis: Forecasting and Control, 1st ed. (Wiley, New York, 1970).

[14] F. Ravelet, A. Chiffaudel, F. Daviaud et al., J. Fluid Mech. 601, 339 (2008).

[15] P.-P. Cortet, A. Chiffaudel, F. Daviaud, and B. Dubrulle, Phys. Rev. Lett. 105, 214501 (2010).

[16] P.-P. Cortet, P. Diribarne, R. Monchaux, A. Chiffaudel, F. Daviaud, and B. Dubrulle, Phys. Fluids 21, 025104 (2009).

[17] P. Cortet, E. Herbert, A. Chiffaudel, F. Daviaud, B. Dubrulle, and V. Padilla, J. Stat. Mech. (2011) P07012.

[18] D. Faranda, F. M. E. Pons, B. Dubrulle, F. Daviaud, B. SaintMichel, É. Herbert, and P.-P. Cortet, Phys. Fluids 26, 105101 (2014).

[19] J. Laval, B. Dubrulle, and S. Nazarenko, Phys. Fluids 13, 1995 (2001).

[20] D. Faranda, B. Dubrulle, and F. M. E. Pons, J. Phys. A: Math. Theor. 47, 252001 (2014). 